REVIEW

\title{
Clinical efficacy and neuroprotective effects of brimonidine in the management of glaucoma and ocular hypertension
}

\author{
Anna Galanopoulos' \\ Ivan Goldberg ${ }^{2}$ \\ 'Senior Visiting Ophthalmologist, \\ South Australian Institute \\ of Ophthalmology and Dept \\ of Ophthalmology and Visual \\ Sciences, University of Adelaide, \\ South Australia; ${ }^{2}$ Clinical Associate \\ Professor, University of Sydney, \\ Australia; Head, Glaucoma Unit, \\ Sydney Eye Hospital, Sydney, Australia, \\ Director, Eye Associates, Sydney, \\ Australia
}

\begin{abstract}
Elevated intraocular pressure (IOP) is a significant risk factor for the development and progression of glaucomatous optic neuropathy, but increasingly we appreciate that non-pressure dependent factors, are key to our understanding of the pathophysiology of these neurodegenerative diseases, that target the retinal ganglion cell. As we try to expand therapy beyond IOP control, medications are being assessed for their neuroprotective abilities. Brimonidine is an effective ocular hypotensive treatment both as a first and second line agent, in the management of glaucoma and ocular hypertension. Brimonidine tartrate $0.2 \%$ is generally safe and well tolerated, with its safety profile further enhanced in the altered formulation brimonidine-Purite ${ }^{\mathrm{TM}} 0.1 \%$. Beyond brimonidine's pressure lowering capacity, laboratory and early clinical evidence supports its neuroprotective potential. We await validation of this in human clinical trials.
\end{abstract}

Keywords: brimonidine, neuroprotection, clinical effectiveness, tolerability

\section{Introduction}

Glaucomatous optic neuropathy (GON) is the manifestation of a group of diseases that progressively destroy retinal ganglion cells and if undetected or untreated, cause visual field loss and functional disability. With increasing prevalence in an ageing population, ${ }^{1,2}$ identification and treatment of patients to prevent visual disability have significant implications for our communities.

As elevated intraocular pressure (IOP) is the most significant risk factor for developing glaucomatous optic neuropathy, and for its progression, its reduction and control have been the mainstays of glaucoma management. However non-pressure-dependent factors also contribute to the pathophysiology of glaucoma. Glaucomatous damage develops in many individuals despite the existence of IOP readings consistently in the usual range (less than $21 \mathrm{mmHg}$ ), ${ }^{2,3}$ conversely in individual patients, despite IOP-reduction, there is continued progression of the GON. ${ }^{4}$

With increased recognition of the neurodegenerative nature of glaucoma, antiglaucoma medications are being assessed for their ability to exert clinical benefits beyond IOP control, specifically their ability to protect retinal ganglion cells and to prevent neuronal cell death (neuroprotection). ${ }^{5}$

Brimonidine tartrate $0.2 \%$ ophthalmic solution (Alphagan ${ }^{\circledR}$, Allergan) is a highly selective alpha-2 adrenergic agonist, which lowers IOP through increased uveoscleral outflow and decreased aqueous humour production. ${ }^{6}$ Beyond this there has been interest in whether brimonidine has neuroprotective effects both in the laboratory and in clinical trials.

\section{Pharmacology and mechanism of action of brimonidine} Brimonidine tartrate, a third generation alpha- 2 adrenergic agonist, has more than 1000-fold selectivity for alpha-2 over alpha- 1 receptors. ${ }^{7}$ This gives it a distinct 
advantage over the first and second generation clonidine and apraclonidine by reducing the risk of systemic side effects, such as systemic hypotension, bradycardia and sedation, as well as reducing the alpha-1 mediated ocular side effects seen more commonly with apraclonidine: conjunctival blanching, mydriasis and eyelid retraction. ${ }^{7}$

On topical application, brimonidine readily penetrates the cornea and results in a reduction of the IOP within 1 hour. Peak effect is achieved within 2 to 3 hours and the trough drug effect occurs approximately 10 to 14 hours after instillation. ${ }^{8}$ In most parts of the world, brimonidine is dosed twice daily and its rapid metabolism and short half-life help to reduce potential cardiovascular and respiratory side effects. ${ }^{7}$ Brimonidine appears to lower IOP by a dual mechanism of action. Initial dosing causes a reduction in aqueous humour production whereas with chronic dosing, the predominant effect is an increase in uveoscleral outflow. ${ }^{8}$

\section{Evidence for neuroprotection}

Neuroprotection in glaucoma refers to the ability to preserve anatomic and functional integrity of the retinal ganglion cells other than by reduction of IOP, thus preserving the visual field. ${ }^{9}$

To evaluate the potential of a pharmacological agent to be useful clinically as a neuroprotectant in glaucoma, 4 criteria have been proposed. ${ }^{10}$

1. There must be specific target receptors in the retina or optic nerve.

2. There must be laboratory evidence that supports it has a mechanism of action that enhances a neuron's resistance to injury.

3. It must be available at the retina or optic nerve at pharmacological concentrations required for a neuroprotective effect after topical dosing, and

4. It must have demonstrated neuroprotective activity in prospective randomized clinical trials.

We will review current evidence that assesses brimonidine with respect to these criteria.

Laboratory evidence has demonstrated alpha- 2 receptors in the retina. Immunohistochemical studies have shown the existence of alpha adrenergic receptors in human, bovine and porcine retina ${ }^{11}$, and in particular in the ganglion cell layer and inner nuclear layer of the rat retina. ${ }^{10}$

Brimonidine requires a concentration of $2 \mathrm{nM}$ to activate alpha-2 adrenergic receptors significantly. ${ }^{12}$ In a clinical study of 17 patients scheduled to undergo pars plana vitrectomy, topical brimonidine $0.2 \%$ was administered twice daily for 5 to 14 days prior to surgery. At the time of surgery an undiluted core of vitreous humour was obtained and brimonidine concentrations, determined. The cohort included phakic, aphakic and pseudo-phakic patients. The mean brimonidine concentration measured in the vitreous was $185 \mathrm{nM}$. As this is well above the $2 \mathrm{nM}$ required for receptor activation, ${ }^{13}$ it seems reasonable to assume that retinal levels are likely to be sufficient to activate the alpha 2 receptors following topical instillation. Phakic eyes demonstrated a lower concentration than did pseudophakic or aphakic eyes, with phakic eyes achieving a mean concentration of $9.3 \mathrm{nM}$ of brimonidine, as compared with $164 \mathrm{nM}$ in aphakic eyes and $256 \mathrm{nM}$ in pseudophakic eyes.

Studies of the effect of brimonidine on experimental animal models of optic nerve injury provide further evidence of its neuroprotective potential. In pre-clinical studies using the chronic ocular hypertensive rat model, the effect of retinal ganglion cell survival was assessed following subcutaneous administration of brimonidine, compared with timolol as the control. In brimonidine-treated eyes, retinal ganglion cell (RGC) death was reduced up to $50 \%$, whereas with timolol, no neuroprotective effect was demonstrated. ${ }^{14}$ Although both drugs have comparable IOP lowering efficacy, neither drug lowered the IOP significantly when administered systemically, suggesting that the beneficial effect seen, directly related to the neuroprotective effect. Further analysis of a rat model of glaucoma, where brimonidine was administered intraperitoneally, revealed attenuation of ganglion cell death independent of IOP reduction. ${ }^{15}$

Retinal injury and brimonidine effects on this have been assessed with two rat models: induction of retinal ischemia and then reperfusion, as well as crush injury to the optic nerve. Administration of brimonidine enhanced retinal ganglion cell survival in both models. ${ }^{16}$ In a similar rat model of retinal ischemia, Donello et al demonstrated preservation of the ERG $b$ wave. ${ }^{17}$

While the mechanism by which this neuroprotection is mediated is not completely understood, alpha- 2 adrenergic receptor stimulation might inhibit signals that trigger the apoptotic cascade in the RGC. ${ }^{18}$

Brimonidine appears to meet the first 3 criteria required of a neuroprotectant; support of clinical trials is necessary to meet the fourth.

An initial randomized controlled study, comparing the effects of brimonidine and timolol on visual field loss, after acute primary angle closure crisis, found no difference in prevalence of visual field defects or rate of field progression between the brimonidine and timolol treated groups. ${ }^{19}$ 
Another randomized double-masked pilot study evaluated the neuroprotective efficacy of brimonidine, when applied to eyes of patients undergoing laser treatment for extrafoveal and juxtafoveal choroidal neovascularization. ${ }^{20}$ Twenty eyes were randomized to receive brimonidine $0.2 \%$ (study group, 11 eyes) or placebo (matched control group, 9 eyes). Brimonidine was instilled twice daily for 4 to 48 hours pre-laser treatment and was continued for one month post- laser. In each group, 2 eyes had severe visual loss from recurrence of the CNV but in the remainder, significant improvement of visual acuity was noted in the study group but not in the control group. The authors proposed that brimonidine improved the visual outcome of laser treated $\mathrm{CNV}$ by protecting the neuroretina against laser-induced collateral damage.

More recently Tsai et al in a prospective unmasked study compared the effects of brimonidine $0.2 \%$ with timolol $0.5 \%$ on retinal nerve fiber layer (RNFL) thickness in ocular hypertensive patients. Using scanning laser polarimetry (GDx) to measure the RNFL, they demonstrated quantitatively less progression of RNFL damage with brimonidine versus timolol $0.5 \%$ in these patients over 12 months. $^{21}$

In a prospective clinical trial, Gandolfi et al compared visual field deterioration rates in patients randomized to either topical brimonidine or to $360^{\circ}$ argon laser trabeculoplasty (ALT). After 18 months of follow up, despite brimonidine reducing IOP less than the ALT, it appeared to be more effective than the laser in reducing field deterioration, in progressing glaucomatous eyes. ${ }^{22}$

Contrast sensitivity has also been shown to improve in brimonidine treated eyes of newly diagnosed glaucoma patients. This effect was independent of IOP lowering effects, supporting a neuroprotective mechanism. ${ }^{23}$

The Low-Pressure Glaucoma Treatment Study (LoGTS) is a large double-masked multi-center, randomized clinical trial comparing the visual outcomes of "low pressure glaucoma" patients randomized to receive either twice daily brimonidine tartrate $0.2 \%$ or timolol maleate $0.5 \%$. One hundred and ninety patients were recruited between 1998 and 2000 and have been followed for at least 4 years. The main study outcome is visual field stability. ${ }^{24,25}$ Results are expected shortly; they are hoped to advance understanding of these patients, where non-pressure dependent factors are more likely to be operative. ${ }^{25}$

\section{Clinical efficacy studies}

Since its introduction in the Australasian market in October 1997, there has been strong growth in the use of brimonidine tartrate $0.2 \%$ both as a first and second line treatment in patients with POAG and ocular hypertension. ${ }^{26}$

The hypotensive efficacy of brimonidine as monotherapy in glaucoma and ocular hypertension has been studied in a many clinical trials and compared with all other classes of topical hypotensive agents.

Initial clinical trials assessed efficacy of brimonidine tartrate $0.2 \%$ compared with timolol maleate $0.5 \%$. One year data in 2 clinical trials found that brimonidine produced similar mean decreases in the IOP at peak compared with timolol, but at trough, the hypotensive effect was less than with timolol. Overall brimonidine demonstrated sustained IOP lowering efficacy comparable with timolol. ${ }^{27,28,29}$ When followed for 3 or 4 years of continuous use, brimonidine maintained an IOP lowering efficacy comparable with timolol and assessment of long term visual field preservation in both groups, was essentially the same. ${ }^{30,31}$

Brimonidine has also been compared with betaxolol $0.25 \%$, which because of its relative cardio-selective adrenergic antagonist activity, offers greater safety from pulmonary side effects, than the non-selective beta-blockers, such as timolol and because of its pharmacokinetics, is also less likely to precipitate cardiac side effects. ${ }^{32,33}$

Judging clinical success after 4 months (IOP reduction, quality of life effects and adverse effects), brimonidine $0.2 \%$ showed a greater clinical success than betaxolol. ${ }^{34}$ A previous clinical trial reported similar results. ${ }^{35}$

A meta-analysis of 3 clinical studies comparing, the efficacy and harm of latanoprost for IOP reduction in glaucoma patients, with brimonidine, found that there was no significant reduction in the mean IOP at 3 months post treatment in the latanoprost group compared with the brimonidine group. Pooled data of adverse events between these two drugs found that patients in the brimonidine group had a significant increase in the number of ocular adverse events (excluding hyperemia). ${ }^{36}$

Three separate studies evaluated the efficacy and safety of brimonidine compared with dorzolamide when used as monotherapy. While there was no overall difference in IOPlowering efficacy, ocular stinging and burning were more common with dorzolamide. ${ }^{37,38,39}$

Several randomized controlled studies have assessed efficacy and safety of brimonidine as adjunctive therapy. When added to ongoing beta-blocker therapy, brimonidine was shown to reduce IOP significantly. ${ }^{40,41}$ Similarly, when added to latanoprost, brimonidine demonstrated significant additional IOP lowering capacity. ${ }^{41,42}$

Clinical trials assessing the efficacy of a fixed combination of brimonidine $0.2 \%$ and timolol $0.5 \%$ (contribution of 
elements studies) showed mean IOP reductions were greater with the fixed combination (Combigan ${ }^{\circledR}$, Allergan) than when the individual agents each were used alone. ${ }^{43}$ The advantage of the fixed combination, ease and convenience of instillation, without drug dilution seen when 2 drops are instilled too close together in time, might result in better patient compliance, thus leading to the higher success rate with this treatment. ${ }^{44}$

These efficacy studies support the versatility of brimonidine as an effective ocular hypotensive agent in patients with glaucoma and ocular hypertension. It is effective as monotherapy and as an adjunctive agent, with the effect being maintained over time.

\section{Safety and tolerability}

Evaluating efficacy and safety of brimonidine $0.2 \%$ after 1,3 and 4 years of use, various studies have supported its overall safety and tolerability: no clinically significant effects on mean heart rate, blood pressure or pulmonary function. ${ }^{28,29,30}$ Unlike timolol, which can induce cardiovascular and pulmonary side effects, ${ }^{45}$ brimonidine generally is not contraindicated in patients with cardiopulmonary disease.

These studies supported a favorable safety profile for brimonidine. The most common systemic side effects included dry mouth, fatigue or drowsiness and headache. ${ }^{28,29,30}$ and the most common ocular side effects seen included ocular irritation, follicular conjunctivitis and blurred vision.

After 1 and 4 years of use, comparative studies between timolol and brimonidine showed equivalent tolerability. There was a slightly greater frequency of burning and stinging from timolol, and of oral dryness and ocular allergies in the brimonidine group. ${ }^{30}$ When brimonidine was compared with betaxolol suspension, both agents were equally well tolerated, ${ }^{32,35}$ although a higher degree of hyperemia has been reported with betaxolol in another clinical trial. ${ }^{46}$

Brimonidine is more stable and less likely to produce an allergic reaction compared with apraclonidine. ${ }^{47}$ However, the major obstacle to long-term use of brimonidine is its propensity to cause ocular allergic reactions, ${ }^{27,28}$ with reported incidence rates of follicular conjunctivitis of $12.7 \%$ (twice daily dosing) and $15.7 \%$ (thrice daily dosing) at 1 year. ${ }^{27}$ For the third year of use, this rate was $4.2 \% .{ }^{48}$ In all cases of allergy, symptoms resolved following discontinuation.

Demographic and clinical factors associated with brimonidine-induced allergy showed that affected individuals had a higher frequency of ocular allergy to all eye drops and a reduction of tear film production. ${ }^{49}$

Allergan Inc., (Irvine, CA) released a reformulated solution of brimonidine, preserved with chlorine dioxide
(Purite $^{\mathrm{TM}}$ ) rather than benzalkonium chloride (BAK), and reduced the concentration of brimonidine to $0.15 \%$. BAK, the most common antimicrobial preservative used in topical ophthalmic preparations, has the potential to accumulate in ocular tissues, and to cause toxicity and/or adverse effects, particularly if there is chronic dosing with multiple glaucoma therapies. ${ }^{50,51}$ In contrast Purite (which is converted to natural tear components when exposed to sunlight) has a wide spectrum of antimicrobial activity and a very low level of toxicity. This formulation (brimonidine $0.15 \%$-Purite), despite having $25 \%$ less active drug than brimonidine $0.2 \%$, has comparable hypotensive efficacy, as well as having $41 \%$ lower incidence of allergic conjunctivitis. ${ }^{52,53}$ Currently this formulation is only available through the special access scheme for patients in Australia.

Brimonidine is absolutely contra-indicated in children; because of a less mature blood:brain barrier, they are susceptible to potentially serious central nervous system side effects including sleepiness, lethargy, bradycardia, hypotension, apnea and coma. ${ }^{54}$

\section{Patient perspectives, acceptability, satisfaction, implications of neuroprotection}

Effectiveness of any medication in the treatment of glaucoma depends on patient adherence to and persistence with the therapeutic regimen. Factors that influence these patient factors include, absence of symptoms from their disease, the number of topical medications, dosing frequency and adverse effects associated with instillation, such as burning and stinging. ${ }^{55,56}$ Quality of life assessments, as scored on the Glaucoma Disability Index, of patients on brimonidine as monotherapy, showed no worsening in the quality of life during the study period. ${ }^{34}$

Fixed combinations of brimonidine $0.2 \%$ /timolol $0.5 \%$ $\left(\right.$ Combigan $\left.^{\circledR}\right)$ have demonstrated significantly lower rates of allergy of up to $50 \%$, when compared with the individual components $^{43,57}$ and greater tolerability, with less burning, when compared with dorzolamide/timolol combinations. ${ }^{58}$ This benefit may be attributable to the lower concentration of BAK that is present in Combigan ${ }^{\circledR}$ compared with the individual components and with the fixed combination of dorzolamide/timolol. ${ }^{43}$ It has also been suggested that the topical beta blocker effects of timolol, may have a protective effect in reducing ocular allergy. ${ }^{59,60}$ Its vasoconstrictive effect and reduced conjunctival hyperemia may be the mechanism by which Combigan ${ }^{\circledR}$ achieves better tolerance and reduced local side effects when applied to the eye. ${ }^{43}$ 
When comparing quality of life measures and patient satisfaction between dosing with brimonidine $2 \%$ and brimonidine-Purite ${ }^{\mathrm{TM}} 0.15 \%$, patients reported superior satisfaction and comfort ratings with the latter formulation. ${ }^{52}$

Overall brimonidine $0.2 \%$ demonstrates hypotensive efficacy, and is tolerated by the majority of patients. Future availability of the Purite $^{\mathrm{TM}}$ formulation will reduce the frequency of topical allergy, without compromising IOP lowering efficacy. Its twice daily dosing, availability in fixed dose combinations, together with its adult safety profile contributes to satisfaction, compliance and persistence with treatment If clinical evidence supports neuroprotective benefits, brimonidine might offer a new therapeutic paradigm, to preserve visual function, beyond IOP reduction.

\section{Conclusions, place in therapy}

Brimonidine tartrate $0.2 \%$, a highly selective alpha-2 adrenergic agonist, is generally an effective and safe ocular hypotensive agent for the long term management of glaucoma and ocular hypertension, whether used as monotherapy, adjunctive therapy or replacement therapy in the treatment of glaucoma. Limiting the success of brimonidine $2 \%$ as a first line agent, is its rate of topical allergy, but this is less common with the brimonidine-Purite ${ }^{\mathrm{TM}}$ formulation.

If clinical studies with brimonidine support laboratory evidence of a neuroprotective effect, our treatment paradigm will shift beyond IOP-reduction, and begin to focus on our ability to preserve visual function for patients, through both neuroprotection and ocular hypotension.

\section{Disclosures}

The authors have no conflicts of interest to disclose.

\section{References}

1. Tunnel vision : The Economic Impact of Primary Open Angle GlaucomaA Dynamic Economic Model. Centre for Eye Research Australia. 2008.

2. Mitchell P, Smith W, Attebo K, et al. Prevalence of open-angle glaucoma in Australia. The Blue Mountains Eye Study. Ophthalmology. 1996;103(10):1661-9.

3. Klein BE, Klein R, Spinsel WE, et al. Prevalence of glaucoma. The Beaver Dam Eye Study. Ophthalmology. 1992;99:1499-504.

4. The AGIS investigators. The Advanced Glaucoma Intervention Study (AGIS):7. The relationship between control of intraocular pressure and visual field deterioration. Am J Ophthalol. 2000;130:429-40.

5. Neuroprotection and Glaucoma; Current Concepts. 2002 Oxford Institiute.

6. Toris CB, Camras CB, Yablonski ME. Acute versus chronic effects of brimonidine on aqueous humor dynamics in ocular hypertensive patients. Am J Ophthalmol. 1999;128(1):8-14.

7. Cantor LB. The evolving pharmacotherapeutic profile of brimonidine, an alpha2-adrenergic agonist, after four years of continuous use. Expert Opin Pharmacother. 2000;1:815-34.
8. Walters TR. Development and use of brimonidine in treating acute and chronic elevations of intraocular pressure, a review of safety, efficacy, dose response and dosing studies. Surv Ophthalmol. 1996;41: S19-S26.

9. Weinreb RN, Levin LA. Is neuroprotection a viable therapy for glaucoma? Arch Ophthalmol. 1999;117:1540-4.

10. Wheeler LA, Gil DW, WoldeMussie E. Role of alph2adrenergic receptors in neuroprotection and glaucoma. Surv Ophthalmol. 2001;45(Suppl 3):S290-294.

11. Bylund DB, Chacko DM. Characterization of alpha2 adrenergic receptor subtypes in human ocular tissue. Ivest Ophthalmol Vis Sci. 1999;40:2299-306,

12. Burke J, Manlapaz C, Kharlamb A, et al. Therapeutic use of a2-adrenoreceptor agonists in glaucoma. In: Lanier SM, Limbird LE (eds). Alpha-Adrenergic Receptors Structure, Function and Therapeutic Implications. Reading, UK, Harwood Academic Publishers; 1996. pp 179-87.

13. Kent A, Nussdorf J, David R, et al. Vitreous concentrations of topically applied brimonidine tartrate $0.2 \%$. Ophthalmology. 2001;108:784-7.

14. WoldeMussie E, Ruiz G, Wijono M, Wheeler LA. Neuroprotection of retinal ganglion cells by brimonidine in rats with laser- induced chronic ocular hypertension. Invest Ophthalmol Vis Sci. 2001:42:2849-55.

15. Hernandez M, Urcola JH, Vecino E. Retinal ganglion cell neuroprortection in a rat model of glaucoma following brimonidine, latanoprost or combined treatments. Exp Eye Res. 2008;86:798-806.

16. Wheeler LA, Lai R, WolenMussie E. From the lab to the clinic: activation of an alpha-2 agonist pathway is neuroprtoective in models of retinal and optic nerve injury. Eur J Ophthalmol. 1999;9(Suppl 1): S17-21.

17. Donello JE, Padillo EU, Webster ML, et al. Alpha(2)-adrenoceptoragonists inhibit vitreal glutamate and aspartate accumulation and preserve retinal function after transient ischaemia. J Pharmacol Exp Ther. 2001;296:216-23.

18. Wheeler LA, Tatton NA, Elstner M, et al. Alpha-2 adrenergic receptor activation by brimondine reduces neuronal apoptosis through Akt (ptrotien Kinase B) dependent new synthesis of BCL-2. Invest Opthalmol Vis Sci. 2001;42:S411.

19. Aung T, Oen FT, Wong HT, et al. Randomised controlled trial comparing the effect of brimonidine and timolol on visual field loss after acute primary angle closure. Br J Ophthalmol. 2004;88(1):88-94.

20. Ferencz JR, Gilady G, Harel O et al. Topical brimonidine reduces collateral damage caused by laser photocoagulation for choroidal neovascularization. Graefes Arch Clin Exp Ophthalmol. 2005;243:877-80.

21. Tsai JC, Chang HW: Comparison of the effects of brimonidine $0.2 \%$ and timolo $0.5 \%$ on retinal nerve fiber layer thickness in ocular hypertensive patients: a prospective, unmasked study. J Ocul Pharmacol Ther. 2005 Dec;21(6):474-82.

22. Gandolfi SA, Sangermani C, Cimino L. et al. Is there a non IOP- related effect of brimonidine on visual field progression in human glaucoma? IOVS, 2004(45) ARVO E-abstract 2298.

23. Evans DW, Hosking SL, Gherghel D, Barlett JD. Contrast sensitivity improves after brimonidine therapy in primary open angle glaucoma: a case for neuroprotection. Br J Ophthalmol. 2003;87(12):1463-5.

24. Krupin T, Liebmann JM, Greenfield DS, Rosenberg LF, Ritch R et al. The Low-pressure Glaucoma Treatment Study (LoGTS) Study design and baseline characteristics of enrolled patients. Ophthalmology. 2005;112:376-85.

25. Krupin T. Special consideration in low tension glaucoma. Can J Ophthalmol. 2007;42:414-7

26 Market Share Data for Australia, from IMS June 2008.

27. Katz LJ. For the Brimonidine Study Groups 1 and 2. Twice-daily brimonidine tartrate $0.2 \%$ vs timolol $0.5 \%$ : 1 year results in glaucoma patients. Am J Ophthalmol. 1999;127:20-6.

28. Schuman JS, Horwitz B Choplin NT, et al. A 1-year study of brimonidine twice daily in glaucoma and ocular hypertension. A controlled, randomized, multicenter clinical trial. Arch Ophthalmol. 1997;115:847-52. 
29. LeBlanc RP; for the Brimonidine Study Group 2. Twelve-month results of an ongoing randomized trial comparing brimonidine tartrate $0.2 \%$ and timolol given twice daily in patients with glaucoma or ocular hypertension. Ophthalmology. 1998;105:1960-7.

30. Melamed S, David R; for the Brionidine Study group 2. Ongoing clinical assessment of the safety profile and efficacy of brimonidine compared with timolol: three year results. Clin Ther. 2000;22:103-11.

31. Cantor LB. The evolving pharmacotherapeutic profile of brimonidine, an alpha 2-adrenergic agonist, after for years of continuous use. Exp Opin Pharmcother. 2000;1(4):815-34.

32. Goldberg I, Goldberg H. Betaxlol eye drops. A clinical trial of safety and efficacy. Aust N Z J Ophthalmol. 1995;23:17-24.

33. Goldberg I. The safety of topical beta-blockers in glaucoma treatment. Med J. Aust 1996;164:49-49.

34. Javitt J, Goldberg I. For the Brimonidine Outcomes Study Group 2. Comparison of the clinical success rates and quality of life of brimonidine tartrate $0.2 \%$ and betaxolol $0.25 \%$ suspension in patients with open-angle glaucoma and ocular hypertension. J Glaucoma. 2000;9:398-408.

35. Serle JB. A comparison of the safety and efficacy of twice daily brimonidine $0.2 \%$ versus Betaxolol $0.25 \%$ in subjects with elevated intraocular pressure. Surv Ophthalmol. 1996;41(9 suppl 1): S39-47.

36. Hodge WG, Lachaine J, Steffensen I. et al. The efficacy and harm of prostaglandin analogues for the IOP reduction in glaucoma patients compared to dorzolamide and brimonidine: a systematic review. Br J Ophthalmol. 2008;92:7-12.

37. Whitson JT, Henry C, Hughes B, et al. Comparison of the safety and efficacy of dorzolamide $2 \%$ and brimonidine $0.2 \%$ in patients with glaucoma or ocular hypertension. J Glaucoma. 2004;13:168-73.

38. Stewart WC, Sharpe ED, Harbin Jr TS, et al. Brimonidine 0.2\% versus dorzolamide $2 \%$ each given three times daily to reduce intraocularpressure. Am J Opthalmol. 2001;29:723-7.

39. Sharpe ED, Day DG, Beischel CJ, et al. Brimonidine purite $0.15 \%$ versus dorzolamide $2 \%$ each given twice daily to reduce intraocular pressure in subjects with open angle glaucoma or ocular hypertension. Br J Ophthalmol. 2004;88:953-6.

40. Simmons ST. Efficacy of brimonidine $0.2 \%$ and dorzolamide $2 \%$ as adjunctive therapy to beta-blockers in adult patients with glaucoma or ocular hypertension. Clin Ther. 2001;23:604-19.

41. Lee DA, Gornbein JA. Effectiveness and safety of brimonidine as adjunctive therapy for patients with elevated intraocular pressure in a large, open label community trial. J Glaucoma. 2001;10:220-6.

42. Konstas AG, Karabatsas CH, Lallos N, et al. 24-hour intraocular pressures with brimonidine purite versus dorzolamide added to latanoprost in primary open-angle glaucoma subjects. Ophthalmology. 2005;112:603-8.

43. Sherwood MB, Craven ER, Chou C et al. Twice-daily $0.2 \%$ brimonidine- $0.5 \%$ timolol fixed-combination therapy vs monotherapy with timolol or brimonidine in patients with glaucoma or ocular hypertension: a 12-month randomized trial. Arch Ophthlmol. $2006 ; 124: 1230-8$
44. Choudri S, Wand M, Shields MB. A Comparison of dorzolamide-timolol combination versus the concomitant drugs. Am J Opthalmol. 200;130:832-3.

45. Everitt DE, Avorn J. Systemic effects of medications used to treat glaucoma. Ann Int Med. 1990;112:120-5.

46. Cantor LB, Hoop J, Katz LJ, et al. For the Alphagan/Betaxolol Clinical Success Study Group. Comparison of the clinical success and quality-of-life impact of brimonidine $0.2 \%$ and betaxolol $0.25 \%$ suspension in patients with elevated intraocular pressure. Clin Ther. 2001;23(7):1032-9.

47. Thompson CD, MacDonald TL, Garst ME, et al. Mechanisms of adrenergic agonist induced allergy:bioactivation and antigen formation. Exp Eye Res. 1997;64(5):767-73.

48. Abelson MB, Chapin M, Batoosingh A, et al. A retrospective examination of drug-induced allergy to Alphagan and a proposal for a new reporting system. Invest Opthalmol Vis Sci. 1999;40(4):S515. Abstract 2718.

49. Manni G, Centofanti M, Sacchetti M, et al. Demographic and clinical factors associated with the development of alphagan induced allergy. J Glaucoma. 2004;13(2):163-7.

50. Berdy GJ, Abelson MB, Smith IM, George MA. Preservative-free artificial tear preparations. Assessment of corneal epithelial toxic effects. Arch Ophthalmol. 1992;110:528-32.

51. Noecker R. Effects of common ophthalmic preservatives on ocular health. Adv Ther. 2001;18:5.

52. Katz LJ. Twelve-month evaluation of brimonidine-purite versus brimonidine in patients with glaucoma or ocular hypertension. J Glaucoma. 2002;11:119-26.

53. Mundorf T, Williams R, Whitcup S et al. A 3 month comparison of efficacy and safety of brimonidine-purite $0.15 \%$ and brimonidine $0.2 \%$ in patients with glaucoma or ocular hypertension. J Ocular Pharmacol Ther. 2003;19:1.

54. Enyedi LB, Freedman SF. Safety and efficacy of brimonidine in children with glaucoma. J AAPOS. 2002;5(5):281-4.

55. Tsai JC, McClure CA, Ramos SE et al. Compliance barriers in glaucoma: A systematic classification. J Glaucoma. 2003;12:393-8.

56. Nordstrom BL, Friedman DS, Mozaffari E et al. Persistence and adherence with topical glaucoma therapy. Am J Ophthalmol. 2005;140:598-606.

57. Motolko MA. Comparison of allergy rates in glaucoma patients receiving brimonidine $0.2 \%$ monotherapy versus fixed-combination brimonidine 0.2\%-timolol 0.5\% therapy. Curr Med Res Opin. 2008 Aug 6. [Epub ahead of print]

58. Chan K, Testa M, McCluskey P. Ocular Comfort of Combination Glaucoma Therapies:Brimonidine 0.2\%/Timolol 0.5\% Compared with Dorzolamide2\%/Timolol 0.5\%. J Ocul Pharmacol Ther. 2007;32(4):372-6.

59. Osborne SA, Montgomery DM, Morris D, McKay IC. Alphagan allergy may increase the propensity for multiple eye-drop allergy. Eye. 2005; 19:129-37.

60. Alvaroado JA. Reduced ocular allergy with fixed-combination 0.2\% brimonidine- 0.5\% timolol. Arch Ophthalmol. 2007;125:717. 